\section{rev Psi}

Revista de Psicología (UNLP)

https://revistas.unlp.edu.ar/revpsi

\title{
¿Se relacionan el temperamento, la asistencia a los jardines maternales y el género con las habilidades sociales durante los primeros años de vida?
}

\author{
Florencia Montenegro ${ }^{1}$ \\ Lucas G. Gago Galvagno $0^{1,2,3}$
}

\section{Correspondencia}

lucas.gagogalvagno@hotmail.com

Filiaciones institucionales

${ }^{1}$ Facultad de Psicología y Relaciones Humanas. Universidad Abierta Interamericana

(Argentina)

\begin{abstract}
${ }^{2}$ Instituto de Investigaciones en Psicología. Laboratorio de Cognición y Políticas Públicas. Universidad de Buenos Aires (Argentina)
\end{abstract}

${ }^{3}$ Consejo Nacional de Investigaciones Científicas y Técnicas (CONICET)

\section{Resumen}

El objetivo de la siguiente investigación fue evaluar las relaciones entre los estilos temperamentales, la asistencia a jardines maternales y el género con las habilidades sociales en los primeros años de vida. La muestra estuvo conformada por 102 infantes de 18 a 48 meses de edad $(M=30,48$ meses; $D S=10,45)$. Se administró a los cuidadores primarios un cuestionario ad-hoc, la escala de Habilidades Sociales adaptada por Lacunza et al. (2009) y el Cuestionario de Conducta de Niñez Temprana (ECBQ-VSF; Rothbart et al., 2001). Se encontraron asociaciones entre los estilos temperamentales y las habilidades sociales, pero no con respecto a la asistencia a los jardines maternales y el género. Se concluye que las diferencias individuales de los infantes estarían contribuyendo a sus capacidades de interacción, y que sería necesario recabar información referida a la calidad de los jardines maternales para hallar variaciones, ya que en este rango etario las conductas sociales aún son muy variables y volátiles.

\section{Palabras clave}

habilidades sociales | temperamento | jardín material | género | infancia

Cómo citar

Montenegro, F. y Gago Galvagno, L. G. (2020). ¿Se relacionan el temperamento, la asistencia a los jardines maternales y el género con las habilidades sociales durante los primeros años de vida? Revista de Psicología, 19(2), 107-121. doi: $10.24215 / 2422572$ XE066

\section{DOI}

10.24215/2422572XE066
Recibido

03 may. 2020

Aceptado

18 oct. 2020

Publicado

24 oct. 2020

Editor

Nicolás Alessandroni | Facultad de Psicología, Universidad Autónoma de Madrid (España)
ISSN

2422-572X

Licencia

(c) Copyright: Montenegro, F. y Gago Galvagno, L. G. Licencia de Cultura Libre $\underline{\text { CC-BY } 4.0}$

Entidad editora

RevPsi es una publicación de la

Facultad de Psicología (Universidad

Nacional de La Plata, Argentina)

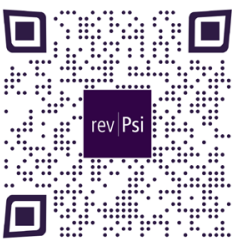

ACCESO ABIERTO DIAMANTE 2 


\section{A frequência do temperamento e do jardim de infância está relacionada às habilidades sociais nos primeiros anos de vida?}

\section{Resumo}

O objetivo da investigação a seguir foi avaliar as relações entre habilidades sociais, estilos temperamentais e jardins de infância nos primeiros anos de vida. A amostra foi constituída por 102 cuidadores primários de lactentes de 18 a 48 meses de idade $(\mathrm{M}=$ 30,48 meses; DP $=10,45)$. Foi aplicado um questionário ad-hoc, a Social Skills Scale adaptada por Lacunza et al. (2009) e o Questionário de comportamento na primeira infância (ECBQ-VSF; Rothbart et al., 2001). Foram encontradas associações entre estilos temperamentais e habilidades sociais, mas não no que diz respeito à frequência do jardim de infância. Concluise que as diferenças individuais dos bebês estariam contribuindo para a sua maneira de interagir e que seria necessário coletar informações sobre a qualidade dos jardins de infância para encontrar variações, uma vez que nessa faixa etária os comportamentos sociais ainda são altamente variáveis e voláteis.

\section{Palavras-chave}

habilidades sociais | temperamento | jardim materno | gênero | infância

\section{Are temperament and kindergarten attendance related to social skills in the first years of life?}

\section{Abstract}

The objective of the following research was to evaluate the relationships between temperamental styles, attendance to daycare and gender with social skills in the first years of life. The sample consisted of 102 infants between 18 and 48 months of age $(\mathrm{M}=30.48$ months; $\mathrm{SD}=10.45)$. The primary caregivers were administered an ad-hoc questionnaire, the Social Skills scale adapted by Lacunza et al. (2009) and the Early Childhood Behavior Questionnaire (ECBQ-VSF; Rothbart et al., 2001). Associations were found between temperamental styles and social skills, but not with regard to kindergarten attendance and gender. It is concluded that the individual differences of infants would be contributing to their interaction capacities, and that it would be necessary to collect information regarding the quality of kindergartens to find variations, since in this age range social behaviors are still highly variable and volatile.

\section{Keywords}

social skills | temperament $\mid$ daycare | gender | childhood 


\section{Aspectos destacados del trabajo}

- La extroversión y el esfuerzo de control se relacionan con mayores niveles de habilidades sociales.

- El esfuerzo de control fue el único estilo temperamental que predice las habilidades sociales.

- La asistencia a jardines maternales no contribuye a modificaciones en las habilidades sociales.

- El género no se relaciona con las habilidades sociales.

Las habilidades sociales infantiles suelen definirse como la capacidad de interactuar con otros de forma efectiva y desarrollar relaciones positivas (Carlson et al., 2019; Fabes, Gaertner y Popp, 2005). Eckerman y Stein (1982) definen las habilidades sociales infantiles como la capacidad para lograr una variedad de resultados sociales importantes aprobados por la sociedad, que conllevan a más cantidad y duración en las interacciones. Esta habilidad es considerada multifacética, ya que son muy variadas las formas en las que se puede expresar en la niñez. En este sentido, las interacciones durante estadíos tempranos del desarrollo pueden ser de tipo lúdico, visual, gestual y verbal (Vandell y Wilson, 1982).

A su vez, Rothbart y Bates (2006) y Van Hecke et al. (2007) incluyen en esta definición la capacidad de integrar los comportamientos del "yo" con los otros durante la interacción, la habilidad del infante de expresar emocionalidad positiva y agradar a los otros y de regular la atención y la reactividad emocional, con el fin de monitorear y corregir posibles errores de interacción y alcanzar así las metas en las actividades que se desarrollan. Es decir, que además de un aspecto comunicativo, las habilidades sociales infantiles estarían fuertemente ligadas a un aspecto emocional (Van Hecke et al., 2007; Weimer y Guajardo, 2005).

A partir del primer año de vida, los infantes comienzan a utilizar gestos dirigidos a objetos y otras personas, ofrecen objetos, imita a otros y cumple solicitudes (Gago Galvagno et al., 2019; Van Hecke et al., 2007). Durante el segundo año de vida y al inicio de la etapa preescolar, el niño comienza a utilizar un repertorio verbal dirigido a la comunicación con otros, estructura la cooperación a partir de nuevos objetos, puede comenzar a comprender las intenciones y la interacción se empieza a dar con otros adultos distintos a sus cuidadores primarios (Durand et al., 2020; Eckerman y Stein, 1982). Estas habilidades sociales primarias son consideradas fundamentales, ya que predicen mayor nivel educativo y de empleo, rendimiento académico, autorregulación, problemas de comportamiento, salud mental y menor actividad criminal y uso de sustancias tanto en la adolescencia como en la adultez 
(Broekhuizen et al., 2016; Jones, Greenbergy Crowley, 2015; Raver et al., 2008). Siendo que se asocian con logros a largo plazo, es importante entender los determinantes que se relacionan con estas habilidades para generar información sobre el tema y en un segundo momento intervenciones específicas (Fabes et al., 2005).

Se ha encontrado en varias investigaciones que los moduladores principales de las habilidades sociales en la infancia temprana son la edad del infante (e.g., Weimer y Guajardo, 2005), los estilos parentales y los tipos de interacción entre cuidador e infante (e.g., Vandell y Wilson, 1982), los tipos de cuidado que se le brindan al infante en las instituciones educativas y el hogar (e.g., Mink, Henning y Aschersleben, 2014) y el temperamento infantil (e.g., Bárrig, Jóa y Alarcón, 2017). Sin embargo, tanto el tipo de cuidado como el temperamento infantil muestran resultados contradictorios $y$ no fueron investigados en infantes de Argentina.

En cuanto al temperamento, Rothbart (2006) afirma que se refiere a las diferencias individuales en los bebés y niños que existen antes de que se desarrollen otros aspectos cognitivos de la personalidad. Este concepto relaciona aspectos genéticos y biológicos que ponen en juego determinadas respuestas afectivas, atencionales y motoras del sujeto (Rothbart, 2011). También tienen un rol en el despliegue de conductas sociales y en el desempeño social (Calkins, 2005).

Rothbart et al. (2001) señalan tres factores del temperamento infantil: a) extraversión, definida por el nivel de actividad, anticipación positiva, búsqueda de sensaciones, sonrisa-risa, impulsividad y placer intenso. Hace referencia al entusiasmo vital de una persona, lo cual se refleja en el nivel de energía y compromiso con el medio (Yap, Allen $y$ Sheeber, 2007), b) esfuerzo de control, que se define por control inhibitorio, atención focalizada, placer de baja intensidad y sensibilidad perceptiva. El cambio o focalización de la atención permite modular la activación emocional, regulando la exposición a un estímulo y los procesos cognitivos relacionados con esas experiencias emocionales (Rothbart, Ahadi y Evans, 2000), lo que permite manejar las conductas manifiestas que se asocian con las emociones (Eisenberg et al., 2000) y c) afectividad negativa, que comprende malestar, miedo, enojo, tristeza, timidez y dificultad para calmarse. Se refiere a una angustia emocional general y determinada susceptibilidad en cuanto a emociones negativas (Rothbart et al., 2000) y se toma como un factor de vulnerabilidad para el desarrollo de trastornos del estado de ánimo y ansiedad (Clark, Watson y Mineka, 1994).

Por otro lado, debido a la alta incidencia de la mujer en el mercado laboral, los infantes asisten cada vez más a jardines maternales y además comienzan a hacerlo a edades más tempranas dentro de Argentina (Marzonetto y Enríquez, 2017). En Argentina, la educación infantil abarca desde los 45 días a los 5 años de edad. Siendo que recién desde los 4 años de edad la educación es obligatoria, el 39\% de los infantes argentinos menores a 3 años asisten a jardines maternales (aumentando un $56 \%$ la matrícula con respecto a los últimos 10 años), mientras que a partir de los 4 años la asistencia asciende al $83 \%$, con la posibilidad de jornada simple o completa para todas las edades (Aulicino, Gerenni y Acuña, 2015; CIPPEC, 2015). Por ende, 
es necesario evaluar cómo se asocia la asistencia a estas instituciones en variables fundamentales para su desarrollo temprano.

En cuanto a las investigaciones previas, en los estudios sobre temperamento y desarrollo de habilidades sociales infantiles se encontró que un temperamento con altos niveles de extroversión y afecto negativo y bajo de esfuerzo de control, puede repercutir en la presencia de problemas de conductas hacia pares y figuras de autoridad (Razza, Martin y Brooks-Gunn, 2012). En la misma línea, existen evidencias de que los niños y niñas con un alto esfuerzo de control, reflejan un mayor manejo de las situaciones y estrategias de afrontamiento (Eisenberg, 2012). En otros estudios, el esfuerzo de control se relaciona de manera positiva con la competencia social y de forma negativa con las conductas agresivas (Berdan et al., 2008; Mink, Henning y Aschersleben, 2014; Miral y Loreto, 2017). Por lo tanto, desarrollar un estilo temperamental tendiente al esfuerzo de control le permitiría al infante adaptarse con mayor facilidad a los cambios que se van produciendo en el medio sin frustrarse (Gago Galvagno et al., 2019). Sin embargo, Bárrig y Alarcón (2017), han encontrado que la extroversión puede predecir problemas emocionales y de agresividad en niños preescolares.

En cuanto a los estudios previos sobre las influencias de los jardines maternales, se ha demostrado un vínculo entre los centros de cuidado infantil de calidad y la cognición con una serie de habilidades cognitivas y socioemocionales que van desde el coeficiente intelectual, el vocabulario, el posterior desempeño en matemáticas, la lectura, preparación escolar y habilidades sociales. Sin embargo, las investigaciones sobre el impacto en las habilidades socioemocionales y temperamentales son principalmente de América del Norte y Europa (Felfe y Zireow, 2018; Fort et al., 2016; NICHD Early Child Care Research Network, 2002). Además, los resultados no son concluyentes, con estudios que muestran un conjunto de efectos que van desde mayores niveles de autorregulación, extroversión y habilidades sociales (Berlinski Galiani y Gertler, 2009; Britto et al., 2017; Cornelissen et al., 2018; Felfe y Lalive, 2018; Felfe, Nollenberger y Rodríguez-Planas, 2014; Noboa-Hidalgo y Urzúa, 2012) a efectos neutrales en habilidades cognitivas y socioemocionales (Cascio, 2009; Gupta y Simonsen, 2010; Lordelo, Chalhub, Guirra y Carvalho, 2007) y hasta efectos negativos de los centros de cuidados en estas habilidades (Felfe y Zireow, 2018, Fort et al., 2017).

Correia-Zanini, Marturano y Fontaine (2018), encontraron que la asistencia a centros educativos durante los primeros años de vida, predecía de forma positiva las habilidades sociales en el nivel primario y promovía las interacciones de los infantes. Sin embargo, para que se presente este efecto, era necesario que asistiesen por lo menos un mes al centro educativo. A resultados similares arribaron Broekhuizen et al. (2016), encontrando que la asistencia a jardines maternales durante el tercer año de vida predecía de forma positiva las habilidades sociales y de forma negativa los problemas comportamentales y el esfuerzo de control. Esta asociación estaba mediada también por la calidad de los jardines maternales (organización en el aula y tipo de intervención de las maestras). Específicamente en Argentina, Aubone et al. (2016), encontraron que la asistencia a jardines maternales aumentaba el nivel 
de habilidades sociales, de cooperación e interacciones en infantes, sin embargo, las muestra fue pequeña $(n=35)$ y sólo se limitó a una comparación de grupos. Sin embargo, la otra investigación realizada en Argentina (Corrales y Schroeder, 2018), no mostró diferencias significativas en habilidades sociales entre los grupos que asistían o no a jardines maternales, trabajando nuevamente con muestras pequeñas $(n=65)$.

En cuanto a las diferencias por género, no se han encontrado en habilidades sociales durante los primeros años (e.g., Aubone et al. 2016; Corrales y Schroeder, 2018). Sería interesante reforzar estos resultados utilizando una muestra de la misma procedencia que la de estas investigaciones, así se podría analizar la robustez de este fenómeno.

En los últimos años se han hecho algunos estudios antes mencionados que relacionan temperamento y habilidades sociales y al infante. Aunque son escasos los estudios analizando estas variables en los primeros años de vida, en población latinoamericana y teniendo en cuenta la asistencia a jardines maternales y el género. Es aquí donde este estudio toma importancia en relación a habilidades sociales, ya que con dicho estudio se puede sumar información relevante sobre el desarrollo social en contexto, el uso de conductas, pautas de desarrollo y la importancia del papel de la institución. A su vez, la relevancia práctica reside en que permitiría generar intervenciones en el equipo docente y psicológico de la institución escolar para generar mayores niveles de habilidades sociales en relación con el temperamento e informar sobre los posibles beneficios del jardín maternal.

El objetivo de la siguiente investigación es: a) relacionar los estilos temperamentales con las habilidades sociales, b) explorar las asociaciones entre la asistencia a jardines maternales y estas habilidades y c) comparar las habilidades sociales en función del género de los infantes. Se espera encontrar que las habilidades sociales se asocien de forma positiva con el esfuerzo de control y extroversión y de forma negativa con el afecto negativo. Por último, no se encontrarán diferencias según el género en las habilidades sociales.

\section{Método}

\section{Diseño}

Se planteó una investigación de tipo cuantitativo, no experimental, transversal y con alcance descriptivo, correlacional, comparativo y predictivo.

\section{Participantes}

Se evaluaron a 102 infantes de 18 a 48 meses $(M=30,48$ meses, $D S=10,45$; femenino= 43; Asiste a jardín= 50), pertenecientes a la Ciudad Autónoma de Buenos Aires y Gran Buenos Aires. De los infantes que asistían, 31 lo hacían a jardines de gestión privada y 19 pública y sólo 6 asistían a jornada completa. La edad promedio de inicio de los infantes fue de 21,32 meses $(D S=7.41$ ). Todas las familias que participaron (98 madres y 4 padres), tenían secundario completo, siendo algunas también de nivel universitario $(n=69)$. 
Se utilizó un tipo de muestreo no probabilístico de tipo intencional y por bola de nieve. Los criterios de selección de la muestra fueron que los infantes posean desarrollo típico, no demuestren antecedentes psiquiátricos o neurológicos, que vivan en Buenos Aires y que naciesen a término.

\section{Instrumentos}

Cuestionario sociodemográfico ad-hoc. Se evaluó la variable jardín maternal a través de los siguientes datos: a) Asistencia a jardín maternal (Sí-No), b) Cantidad de meses que asistió al jardín maternal y c) Horas mensuales que asiste al jardín maternal. A su vez, se indagó sobre la edad en meses y género del infante y el nivel educativo de los padres.

Escala tipo Screening de Habilidades Sociales para cuidadores (adaptada por Lacunza, Castro Solano y Contini, 2009). La escala utilizada en esta investigación fue creada para cuidadores de niños de 2 a 5 años. La evaluación consiste en medir el grado de Habilidades Sociales, entendidas como las capacidades o destrezas sociales específicas requeridas para ejecutar competentemente una tarea interpersonal. Este constructo se midió a través de 12 ítems que indagaron, a través de afirmaciones, si el infante realiza interacciones verbales y no verbales (tanto pasivas como activas) y si es amable con sus pares y adultos. Se trata de una escala unidimensional que conforma un puntaje total a partir de tres opciones de respuesta: 1) Nunca, 2) Algunas veces y 3) Frecuentemente. Por ende, el rango de puntajes posible era de 12 a 36, siendo el puntaje promedio entre 20 y 26 . El Alpha de Cronbach para el factor de habilidades sociales fue de .73 .

Cuestionario de Conducta de Niñez Temprana versión resumida (ECBQ-VSF, versión latinoamericana, de Rothbart et al., 2001). Para niños de 18 a 36 meses, que evalúa la conducta emocional de los niños desde el punto de vista de los cuidadores. La conducta es clasificada siguiendo una escala Likert de 8 puntos que significa 1) Nunca, 2) Casi nunca, 3) Menos de la mitad del tiempo, 4) Aproximadamente la mitad del tiempo, 5) Más de la mitad del tiempo, 6) Casi siempre, 7) Siempre y 8) No sucedió (no se asigna puntaje). Esta prueba consta de 36 ítems y cada subescala está formada por 12 ítems. El puntaje final de estas se forma a partir del promedio de los ítems, siendo de 1 a 7 el rango de puntaje. Las escalas son las de extraversión, afectividad negativa y esfuerzo de control; evalúa la capacidad de inhibir o suprimir respuestas dominantes. Involucra procesos cognitivos tales como cambio atencional, atención focalizada, control inhibitorio, baja intensidad de la sensación de placer y sensibilidad perceptual. Siendo que parte de la muestra superó el rango etario propuesto para este instrumento, se calcularon el índice de Alpha para los infantes de 18 a 36 meses y para los de 36 a 48 meses. En el primer grupo, se obtuvo un Alpha de .72, .79 y .81, mientras que el segundo grupo uno de $.66, .69$ y .70. para afecto negativo, extroversión y esfuerzo de control respectivamente, lo cual demuestra la ausencia de diferencias importantes y es aceptable para ambas muestras (Putnam et al., 2010). 


\section{Procedimiento}

Las baterías fueron administradas de forma voluntaria y anónima a los cuidadores primarios de los infantes mediante Google Formularios. Los investigadores proporcionaron mails de contacto en los cuestionarios, que ante la duda por parte de los participantes acerca de alguna pregunta o ítem, fueron consultados.

Al comienzo de los formularios se explicó a los participantes los objetivos de la investigación y acerca de los cuestionarios. Luego debían aceptar su participación y completar los cuestionarios. Los mismos fueron aplicados siempre en el mismo orden para establecer un control por equiparación y repartir equitativamente el efecto aprendizaje y fatiga en el total de la muestra.

La muestra fue reclutada a través de grupos de Facebook y WhatsApp, a las figuras de cuidado primario de los infantes, quienes a su vez enviaron este formulario a distintos jardines maternales.

\section{Análisis de datos}

Los datos recopilados fueron procesados a través del software IBM-SPSS (Statistical Package for the Social Sciences), versión 24. Se realizó una prueba de normalidad de Kolmogórov-Smirnov y la prueba de Levene para calcular homogeneidad de varianzas, a modo de indagar la distribución de las variables. Siendo que la mayoría de las variables se distribuyeron de forma normal, que las varianzas fueron homogéneas $(p>.05)$ y que el tamaño de la muestra fue mayor a 30, se determinó utilizar estadísticos paramétricos para realizar los análisis posteriores.

Se procedió, en primer lugar, a realizar la estadística descriptiva para las variables medidas. Luego se aplicó una prueba de correlación parcial $\mathrm{R}$ de Pearson controlando la variable edad (ya que se encontró una asociación entre esta y las habilidades sociales, $r=.37, p=.001$ ), para probar la hipótesis de asociación entre el temperamento y las habilidades sociales. La misma prueba se aplicó para las asociaciones entre habilidades sociales y los meses y horas de asistencia a jardín maternal, pero analizando únicamente a los sujetos que asistían al jardín $(n=50)$.

Luego, al encontrarse asociaciones entre el temperamento y las habilidades sociales, se realizó una regresión lineal múltiple por bloques, examinando si los estilos temperamentales (extroversión, afecto negativo y esfuerzo de control) y sus interacciones predecían a las habilidades sociales. Se controló la variable edad de los infantes. Por último, se realizó la prueba de ANOVA para evaluar la diferencia de las habilidades sociales en función del género, insertando la variable edad de los infantes como covariable. 


\section{Resultados}

\section{Estadística descriptiva}

Se encontraron puntajes totales moderados para las variables de habilidades sociales (Lacunza et al., 2009). El estilo temperamental de extroversión fue el que más puntaje obtuvo, siendo mayor al puntaje promedio encontrado en infantes de la misma edad (Gago Galvagno et al. 2019; Rothbart et al., 2001). Esto podría deberse a la tendencia de los padres a sobrevalorar los aspectos positivos de sus infantes en los reportes parentales (Freund, 2019), o también a que la muestra poseía una media de edad cercana a los tres años de vida. Se encontró además que había, para esta muestra, una alta variabilidad en la cantidad de horas por mes y la cantidad de meses que hacía que asistían a los jardines maternales, ya que algunos infantes asistían a jornada completa y la edad de ingreso varió entre los sujetos. La estadística descriptiva se presenta en la Tabla 1.

\section{Asociaciones entre habilidades sociales y estilos temperamentales}

Se encontró una correlación positiva entre extroversión y las habilidades sociales, siendo que a medida que aumenta la propensión a la búsqueda de sensaciones y de explorar el medio, también lo hicieron las habilidades de interacción del infante. Además, hubo una asociación positiva entre el esfuerzo de control y las habilidades sociales, siendo que a medida que incrementaba el estilo temperamental referido a refrenar los impulsos e inhibir comportamientos, aumentaban las habilidades sociales. El resumen de los resultados se muestra en la Tabla 2.

\section{Predicción de las habilidades sociales a través del temperamento}

Para las habilidades sociales, el modelo general fue significativo, indicando y explicando el $21 \%$ de la variabilidad en las habilidades. Solo el esfuerzo de control se relacionó significativamente con las habilidades sociales en este modelo, indicando que a medida que el esfuerzo de control aumentaba, también lo hicieron las habilidades sociales. No se encontraron resultados significativos en cuanto a estas interacciones $(p>.05)$. En la Tabla 3 se detallan los resultados encontrados.

Tabla 1. Estadística descriptiva de las variables medidas

\begin{tabular}{lrrrr}
\hline \multicolumn{1}{c}{ Medidas } & \multicolumn{1}{c}{ M(DS) } & \multicolumn{1}{c}{ 95\%IC } & \multicolumn{1}{c}{ Rango } & \multicolumn{1}{c}{ n } \\
\hline Habilidades sociales & $28.71(4.35)$ & {$[27.85,29.56]$} & $17-36$ & 102 \\
Extroversión & $5.30(0.80)$ & {$[5.14,5.46]$} & $2.33-6.90$ & 102 \\
Esfuerzo de control & $4.97(0.85)$ & {$[4.80,5.14]$} & $2.58-7$ & 102 \\
Afecto negativo & $3.35(1.01)$ & {$[3.15,3.55]$} & $1.30-6$ & 102 \\
Meses que asiste $^{*}$ & $14.40(10.51)$ & {$[11.33,1747]$} & $1-54$ & 50 \\
Horas que asiste (por mes) $^{*}$ & $88.26(42.97)$ & {$[76.05,100.47]$} & $0-180$ & 50 \\
Edad en meses $^{3}$ & $30.48(3.45)$ & {$[28.37,32.59]$} & $18-48$ & 102 \\
\hline
\end{tabular}

*Se describió para el grupo de infantes que asistían a jardín. Nota. IC: Intervalo de confianza. 
Tabla 2. Correlaciones parciales entre las habilidades sociales con los estilos temperamentales y horas-meses que asiste

\begin{tabular}{|c|c|c|c|c|c|c|}
\hline Variables & 1. & 2. & 3. & 4. & 5. & 6. \\
\hline 1. Habilidades sociales & - & -.12 & $.23^{\star}$ & $.34^{\star *}$ & .14 & .15 \\
\hline 2. Afecto negativo & & - & -.04 & $-.30^{\star *}$ & .02 & -.08 \\
\hline 3. Extroversión & & & - & $.41^{\star *}$ & -.02 & -.03 \\
\hline 4. Esfuerzo control & & & & - & -.17 & -.12 \\
\hline 5. Horas que asiste & & & & & - & $.64^{\star *}$ \\
\hline 6. Meses que asiste & & & & & & - \\
\hline
\end{tabular}

Nota. Las correlaciones de Pearson fueron reportadas para todas las variables. ${ }^{*} p<.05 .{ }^{* *} p<.01$

\section{Asociaciones entre habilidades sociales y asistencia a jardín}

No se encontraron asociaciones entre la cantidad de meses que los infantes asistieron a jardín y las habilidades sociales $(p>.05)$. Tampoco se encontraron asociaciones entre la cantidad de horas mensuales que los infantes asistieron a jardín y estas habilidades $(p>.05)$. El resumen de los resultados se muestra en la Tabla 2.

No se encontraron diferencias en las habilidades sociales en función de la asistencia o no a jardines maternales $(p>.05)$.

\section{Comparación de grupos por género y asistencia a jardín}

No se encontraron diferencias en las habilidades sociales en función del género $(p>.05)$.

\section{Conclusión}

El objetivo de la siguiente investigación fue: a) relacionar los estilos temperamentales con las habilidades sociales, b) explorar las asociaciones entre la asistencia a jardines maternales y estas habilidades y c) comparar las habilidades sociales en función del género de los infantes. Los resultados obtenidos demuestran que hay una correlación positiva entre las variables habilidades sociales con extroversión y esfuerzo de

Tabla 3. Predicción de las habilidades sociales a través de las subdimensiones del temperamento

\begin{tabular}{lccccc}
\hline \multicolumn{1}{r}{ Temperamento } & F & R2 & B & $\boldsymbol{\beta}$ & \multicolumn{2}{c}{ p } \\
\hline Edad & 15.19 & .123 & .147 & .363 & .001 \\
\hline Edad & 7.95 & .212 & .135 & .333 & .001 \\
Extroversión & & & .575 & .106 & .283 \\
Afeco negativo & & & -.259 & -.060 & .521 \\
Esfuerzo de control & & & 1.310 & .256 & .014 \\
\hline
\end{tabular}


control. Además, se encontró que la cantidad de meses y horas que asisten los niños al jardín y la asistencia o no a estas instituciones no se relacionaron con las habilidades sociales. Por último, no se encontraron diferencias en las habilidades sociales según el género.

En cuanto a los resultados entre las asociaciones de temperamento y habilidades sociales, los mismos van de la mano con los de estudios previos (Berdan et al., 2008; Eisenberg, 2012; Mink, Henning y Aschersleben, 2014), en donde se resaltó la importancia que tienen el esfuerzo de control para las habilidades sociales. El control voluntario está relacionado con la inhibición de comportamientos disruptivos o impulsivos, lo que permite controlar la atención focalizada y modular la activación emocional (Rothbart et al., 2000, 2001). Las destrezas sociales y conductas asertivas son requeridas para ejecutar competentemente una tarea con características de interacción interpersonal (Fabes, Gaertner y Popp, 2005). Es por ello que el control voluntario repercute fuertemente en las destrezas sociales ya que permite tener conductas positivas y adecuados vínculos interpersonales. En otras palabras, el esfuerzo de control tiene como característica, controlar de manera voluntaria la atención, poner en marcha la conducta, especialmente cuando el niño no está interesado en hacerlo.

En cuanto a las asociaciones positivas con extroversión, otras investigaciones que demuestran que los infantes que poseen mayores niveles de búsqueda de sensaciones y propensión a la interacción y más capacidad de regular los comportamientos ante estímulos ambientales, poseen más habilidad para socializar con el medio en el que se encuentran (Razza, Martin y Brooks-Gunn, 2012; Wagers y Kiel, 2019), ya que aumentan la cantidad de búsqueda de interacciones que el infante activamente ejecuta (Rothbart et al., 2001). La falta de contribución en el modelo de regresión podría deberse a que para que el temperamento infantil contribuya de forma positiva al desarrollo cognitivo, este tiene que estar acompañado de altos niveles de esfuerzo de control y bajos de afectividad negativa (Felfe y Zireow, 2018; Wagers y Kiel, 2019).

En cuanto a la falta de asociación entre la asistencia a jardines y las habilidades sociales y temperamentales infantiles, coinciden con investigaciones previamente realizadas (Corrales y Schroeder, 2018; Felfe y Zireow, 2018, Fort et al., 2017), sin embargo, se contrapone con otras (Aubone et al., 2016; Britto et al., 2017; Cornelissen et al., 2018). En este sentido, Reyna y Brussino (2015) afirman que niños en su primera infancia no manifiestan en general diferencias en comportamiento social, ya que aún se encuentra en desarrollo en los primeros años, por ende los comportamientos en este rango son más volátiles y menos estables, siendo que es un período del desarrollo significativo y veloz. Lo mismo encontraron Seguel et al. (2012), siendo que en los primeros tres años de vida la asistencia a dichos centros no influye en gran medida el desarrollo de los niños a esa edad. Esto va de la mano con la relación positiva entre las habilidades sociales y la edad en meses, que se podría deber a la maduración de la atención, que permite favorecer las habilidades de autorregulación como planificar la conducta deseada, inhibir 
respuestas inapropiadas y focalizar la atención (Kochanska, Murray y Harlan, 2000; Rothbart et al., 2001). Por ende, el infante puede interactuar con otros de forma más adecuada, ya que cuenta con capacidades de autorregulación y lenguaje más desarrolladas (Reyna y Brussino, 2015). Es necesario resaltar además que al ser medidas estas variables mediante reportes parentales, los padres de los niños podrían estar sesgando los resultados (Freund et al., 2019). Es necesario subrayar que en esta investigación solo se midieron variables cuantitativas como tiempo de asistencia en días y meses, siendo que quizá la calidad de las instituciones evaluadas no era lo suficientemente eficaz para producir modificaciones en estas habilidades.

En cuanto al género, no se encontraron diferencias, lo que refuerza los resultados encontrados en Argentina en infantes y preescolares (Aubone et al., 2016; Reyna y Brussino, 2015).

Respecto a las limitaciones, la muestra fue homogénea (infantes de la Ciudad Autónoma y Gran Buenos Aires) y no siguió un muestreo de tipo probabilístico, lo cual dificulta la generalización de los resultados. Además, el rango de edades es bastante amplio, lo cual pudo haber generado variaciones muy grandes debido a que en ese período se dan cambios muy acentuados en un intervalo corto de tiempo. También, se puede presentar como limitación, el no haber tenido en cuenta variables extrañas derivadas de los reportes parentales y la variabilidad en cuanto a la calidad de las diferentes instituciones educativas, que posiblemente sesgaron los resultados. Por último, el haber realizado un estudio transversal no permite visualizar el desarrollo de estas habilidades en cada uno de los niños.

Para futuras investigaciones sería beneficioso ampliar la muestra y reclutarla de diferentes provincias de Argentina. De igual manera, se recomendaría realizar muestreo de tipo probabilístico, basados en una medición directa de los comportamientos de los infantes y con rangos etarios más acotados y teniendo en cuenta la calidad de los diferentes centros educativos. Por último, realizar un estudio de tipo longitudinal, para poder comparar a una misma muestra a lo largo del tiempo y observar así las líneas de desarrollo de los infantes. Esto llevaría, en un segundo momento, a generar intervenciones específicas que tengan en cuenta el desarrollo de los infantes y la incidencia de los factores sociales e individuales, para promover sus habilidades en los primeros años de vida. 


\section{Referencias}

Aubone, N. S., Franco, P., Mustaca, A. E. (2016). Habilidades sociales en niños y su relación con el jardín maternal. ConCiencia EPG, 1(2), 57-71. HTTPS://DOI.ORG/10.32654/CONCIENCIAEPG.1-2.5

Aulicino, C., Gerenni, F., y Acuña, M. (2015). Primera infancia en Argentina: políticas a nivel nacional. Documento de Trabajo No143. CIPPEC Políticas Públicas.

Bárrig Jó, P., y Alarcón Parco, D. (2017). Temperamento y competencia social en niños y niñas preescolares de San Juan de Lurigancho: un estudio preliminar. Liberabit, 23(1), 75-88. HTTPS://DOI.ORG/10.24265/LIBERABIT. 2017. V23N1.05

Berdan, L. E., Keane, S. P., y Calkins, S. D. (2008). Temperament and externalizing behavior: social preference and perceived acceptance as protective factors. Developmental Psychology, 44(4), 957968. HTTPS://DOI.ORG/10.1037/0012-1649.44.4.957

Berlinski, S., Galiani, S. y Gertler, P. (2009). The effect of pre-primary education on primary school performance. Journal of Public Economics, 93(1-2), 219-234. HTTPS://DOI.ORG/10.1016/J. JPUBEC0.2008.09.002

Blanco, A. (1981). Evaluación de las habilidades sociales. En R. Fernández Ballesteros y J. A. Carrobles (Eds.), Evolución conductual: metodología y evaluación (pp. 568). Pirámide.

Britto, P. R., Lye, S. J., Proulx, K., Yousafzai, A. K., Matthews, S. G., Vaivada, T., ... y MacMillan, H. (2017). Nurturing care: promoting early childhood development. The Lancet, 389, 91-102. HTTPS://DOI.ORG/10.1016/so140-6736(16)31390-3

Broekhuizen, M. L., Mokrova, I. L., Burchinal, M. R., Garrett-Peters, P. T., \& Family Life Project Key Investigators. (2016). Classroom quality at pre-kindergarten and kindergarten and children's social skills and behavior problems. Early Childhood Research Quarterly, 36, 212-222. HTTPS://DOI.ORG/10.1016/J.ECRESQ.2016.01.005

Calkins, S.D. (2005). El temperamento y su impacto en el desarrollo infantil: Comentarios sobre Rothbart, Kagan y Eisenberg. En R.E. Tremblay, M. Boivin y R. Peters (Eds.), Enciclopedia sobre el desarrollo de la primera infancia. Nueva York. Wiley. HTTPs://DOI.ORG/10.35376/10324/10385

Carson, V., Lee, E. Y., Hesketh, K. D., Hunter, S., Kuzik, N., Predy, M., ... y Hinkley, T. (2019). Physical activity and sedentary behavior across three time-points and associations with social skills in early childhood. BMC Public Health, 19(1), 1-8.
CIPPEC (2015). Tasa de matriculación por edad simple, 0 a 5 años, en Argentina. DiNIEE-MED.

Clark, L. A., Watson, D., y Mineka, S. (1994). Temperament, personality, and the mood and anxiety disorders. Journal of Abnormal Psychology, 103(1), 103-116. HTTPs://DOI. ORG/10.1037/0021-843X.103.1.103

Cochet, H. y Byrne, R. W. (2016). Communication in the second and third year of life: Relationships between nonverbal social skills and language. Infant Behavior and Development, 44, 189-198.

Cornelissen, T., Dustmann, C., Raute, A. y Schönberg, U. (2018). Who benefits from universal child care? Estimating marginal returns to early child care attendance. Journal of Political Economy, 126, 2356-2409. HTTPS://DOI.ORG/10.1086/699979

Corrales, P. V. y Schroeder, M. B. (2018). Habilidades sociales en niños/as escolarizados de cuatro años que asistieron y no asistieron a jardín maternal (Tesis de Licenciatura). Buenos Aires: Universidad Católica Argentina. HTTPs://DOI. ORG/10.26422/AUCOM.2017.0602.URE

Correia-Zanini, M. R. G., Marturano, E. M. y Fontaine, A. M. G. V. (2018). Effects of early childhood education attendance on achievement, social skills, behaviour, and stress. Estudos de Psicologia, 35(3), 287-297. HTTPS://DOI. ORG/10.1590/1982-02752018000300007

Durand, M., Martínez, M., Gago Galvagno, L. y Elgier, A. (2020). El desarrollo de la comunicación preverbal y verbal temprana. La importancia del juego. Revista Iberoamericana de Psicología, 13(1), 23-32. HTTPS://DOI.ORG/10.33881/20271786.RIP. 13103

Eckerman, C. O. y Stein, M. R. (1982). The toddler's emerging interactive skills. In Peer relationships and social skills in childhood (pp. 41-71). Springer.

Eisenberg, N. (2012). Temperamental effortful control (self-regulation). Encyclopedia on Early Childhood Development. Wiley.

Eisenberg, N., Fabes, R. A., Guthrie, I. K., \& Reiser, M. (2000). Dispositional emotionality and regulation: their role in predicting quality of social functioning. Journal of Personality and Social Psychology, 78(1), 136. HTTPs://DOI. ORG/10.1037/0022-3514.78.1.136

Fabes R.A., Gaertner B.M. y Popp T.K (2005). Getting along with others: Social competence in early childhood. En K. McCartney y D. Phillips (Eds.), Blackwell handbook of early childhood development. Blackwell Publishing. 
Felfe, C. y Lalive, R. (2018). Does early child care affect children's development? Journal of Public Economics, 159, 33-53. HTTPS://DOI.ORG/10.1016/J. JPUBEC0.2018.01.014

Felfe, C. y Zierow, L. (2018). From dawn till dusk: Implications of full-day care for children's development. Labour Economics, 55, 259-281. HTTPS://DOI.ORG/10.1016/J.LABECO.2018.10.006

Felfe, C., Nollenberger, N. and Rodríguez-Planas, N. (2014). Can't buy mommy's love? Universal childcare and children's long-term cognitive development. Journal of Population Economics, 7(2), 14-27. HTTPS://DOI.ORG/10.1007/s00148-014$0532-\mathrm{X}$

Fort, M., Ichino, A. y Zanella, G. (2016). Cognitive and non-cognitive costs of daycare 0-2 for girls. ZA Discussion Papers, 9756. HTTPs://DoI. ORG/10.2139/SSRN.2737370

Fort, M., Ichino, A. y Zanella, G. (2017). The cognitive cost of daycare 0-2 for children in advantaged families. Reporte técnico. Mimeo.

Freund, J. D. (2019). Early temperament in parental report and scientific observation. Early Child Development and Care, 189(14), 2318-2333. HTTPS://DOI.ORG/10.1080/03004430.2018.1450252

Frick, M. A., Bohlin, G., Hedqvist, M., \& Brocki K. C. (2019). Temperament and cognitive regulation during the first 3 years of life as predictors of inattention and hyperactivity/ impulsivity at 6 years. Journal of Attention Disorders, 23(11), 1291-1302. HTTPS://DOI. ORG/10.1177/1087054718804342

Gago Galvagno, L. G., De Grandis, M. C., Clerici G. D., Mustaca, A. E., Miller, S. E. y Elgier, A. M. (2019). Regulation during the second year: Executive function and emotion regulation links to joint attention, temperament and social vulnerability in a Latin American sample. Frontiers in Psychology, 10, 1473. HTTPS://DOI. ORG/10.3389/FPSYG.2019.01473

Gupta, N. D. y Simonsen, M. (2010). Non-cognitive child outcomes and universal high quality child care. Journal of Public Economics, 94, 30-43. HTTPS://DOI.ORG/10.1016/J.JPUBECO.2009.10.001

Jones, D. E., Greenberg, M., y Crowley, M. (2015). Early social-emotional functioning and public health: the relationship between kindergarten social competence and future wellness. American Journal of Public Health, 105(11), 2283-2290.

Kochanska, G., Murray, K. y Harlan, E.T. (2000) Effortful control in early childhood: Continuity and change, antecedents, and implications for social development. Developmental Psychology, 36(2), 220232. HTTPS://DOI.ORG/10.1037/0012-1649.36.2.220
Lacunza, A. B., Solano, A. C. y Contini, N. (2009). Habilidades sociales preescolares: una escala para niños de contextos de pobreza. Revista de Psicología, 27(1), 4-28.

Lordelo, E. D. R., Chalhub, A. A., Guirra, R. C., \& Carvalho, C. S. (2007). Context and cognitive development: attendance to day care center and evolution of mental development. Psicologia: Reflexão e Crítica, 20, 324-334. HTTPs://DOI. ORG/10.1590/s0102-79722007000200019

Marzonetto, G., \& Enríquez, C. R. (2017). El desafío de las capacidades estatales para construir un sistema nacional de cuidados en Argentina. En XXXV International Congress Latin American Studies Association. Lima, Perú.

Mink, D., Henning, A. y Aschersleben, G. (2014). Infant shy temperament predicts preschooler's theory of mind. Infant Behavior and Development, 37(1), 66-75. HTTPS://DOI.ORG/10.1016/J. INFBEH.2013.12.001

Miral, A., y Vera-Nuñez, L. (2017). Control esforzado: componente regulatorio del temperamento y sus implicancias en el desarrollo socio emocional de los niños. Revista Chilena de Neuropsicología, 12(1), 24-28.

Moreno, M. y Cubero, R. (1990). Relaciones sociales: familia, escuela, compañeros. Años preescolares. Alianza.

NICHD Early Child Care Research Network (2000). The relation of child care to cognitive and language development. Child Development, 71, 960-980. HTTPS://DOI.ORG/10.1111/1467-8624.00202

NICHD Early Child Care Research Network. (2002). Child-care structure, process and outcome: Direct and indirect effects of childcare quality on young children's development. Psychological Science, 13(3), 199-206. нттрs:// DOI.ORG/10.1111/1467-9280.00438

Noboa-Hidalgo, G. E. y Urzua, S. S. (2012). The effects of participation in public child care centers: Evidence from Chile. Journal of Human Capital, 6, 1-34. HTTPS://DOI.ORG/10.1086/664790

Putnam, S. P., Jacobs, J., Gartstein, M. A. y Rothbart, M. K. (2010). Development and assessment of short and very short forms of the Early Childhood Behavior Questionnaire. Poster presentado en International Conference on Infant Studies. HTTPS://DOI.ORG/10.1037/T62181-000

Raver, C. C., Jones, S. M., Li-Grining, C. P., Metzger, M., Champion, K. M. y Sardin, L. (2008). Improving preschool classroom processes: Preliminary findings from a randomized trial implemented in head start settings. Early Childhood Research Quarterly, 23(1), 10-26. 
Razza, A., Martin, J.y Brooks-Gunn, R. (2012). Anger and children's socioemotional development: Can parenting elicit a positive side to a negative emotion? Journal of Child and Family Studies, 21(5), 845-856. HTTPS://DOI.ORG/10.1007/S10826011-9545-1

Reyna, C. \& Brussino, S. (2015). Diferencias de edad y género en comportamiento social, temperamento y regulación emocional en niños argentinos. Acta Colombiana de Psicología, 18(2), 51-64. HTTPs:// DOI.ORG/10.14718/ACP.2015.18.2.5

Rothbart, M. (2011). Becoming who we are. Temperament and personality in development The Guilford Press.

Rothbart, M. K. y Bates, J. E. (2006). Temperament. En W. Damon y R. Lerner (Eds.), Handbook of child psychology. Vol 3: Social, emotional, and personality development (pp. 99-166). Wiley.

Rothbart, M. K., Ahadi, S. A. y Evans, D. E. (2000). Temperament and personality: origins and outcomes. Journal of Personality and Social Psychology, 78(1), 122. HTTPs://DOI. ORG/10.1037/0022-3514.78.1.122

Rothbart, M. K., Ahadi, S. A., Hershey, K. L. y Fisher, P. (2001). Investigations of temperament at three to seven years: The Children's Behavior Questionnaire. Child Development, 72(5), 1394 1408. HTTPS://DOI.ORG/10.1111/1467-8624.00355

Vandell, D. L. y Wilson, K. S. (1982). Social interaction in the first year: Infants' social skills with peers versus mother. En Peer relationships and social skills in childhood (pp. 187-208). Springer.
Vaughan Van Hecke, A., Mundy, P. C., Acra, C. F., Block, J. J., Delgado, C. E., Parlade, M. V., ... y Pomares, Y. B. (2007). Infant joint attention, temperament, and social competence in preschool children. Child Development, 78(1), 53-69.

Wagers, K. B. y Kiel, E. J. (2019). The influence of parenting and temperament on empathy development in toddlers. Journal of Family Psychology, 33(4), 391. HTTPs://DOI.ORG/10.1037/ FAM0000505

Weimer, A. A. y Guajardo, N. R. (2005). False belief, emotion understanding, and social skills among Head Start and non-Head Start children. Early Education and Development, 16(3), 341-366.

Willis, E. (2016). An empathetic beginning in education: exploring the prospects of selfregulation skills on pro-social behaviour in the early childhood environment. Early Child Development and Care, 186(4), 662-670. нттPs:// DOI.ORG/10.1080/03004430.2015.1045422

Yap, A. y Sheeber, L. (2007). Uso de un marco de regulación emocional para comprender el papel del temperamento y los procesos familiares en riesgo de trastornos depresivos en adolescentes. Clinical Child and Family Psychology Review, 10(2), 180-96.

Zabalza, M. A. (1997) Calidad en la educación infantil. Los diez aspectos claves de una educación infantil de calidad. Narcea. 